\title{
Circuit Theoretic Classification and Performance Comparison of Parallel-Connected Switching Converters
}

\author{
Yuehui Huang and Chi K. Tse \\ Department of Electronic and Information Engineering, The Hong Kong Polytechnic University, Hong Kong
}

\begin{abstract}
This paper studies the various paralleling styles for dc/dc switching converters from a circuit theoretic viewpoint. The purpose is to examine all possible paralleling structures and control configurations, allowing simple and direct comparison of the characteristics and limitations of different paralleling schemes. In the paper, a circuit theoretic classification of parallel connected converters is described firstly. Converters are modeled as current sources or voltage sources in the classification, and their connection possibilities are categorized systematically into three basic types. Then, control arrangements are classified according to the presence of current-sharing and voltage-regulation loops. Moreover, comparison is made for all the schemes in terms of their performances in current sharing and voltage regulation. Finally, an experiment prototype is built to validate the analysis.
\end{abstract}

\section{INTRODUCTION}

Paralleling of power converters has become a popular approach to constructing power supplies for high-current highpower applications, with high degree of flexibility, maintainability, reliability and ease of expansion. One basic objective of parallel-connected converters is to share the load current among the constituent converters. To do this, some form of control has to be used to equalize the currents in the individual converters. A variety of approaches, with varying levels of complexity and current-sharing performance, have been proposed in the past two decades [1]. In general, methods for paralleling $\mathrm{dc} / \mathrm{dc}$ converters are described in terms of connection styles, control configurations and feedback functions. Comparative studies have also been made to selected paralleling configurations [1]-[2]. However, most of these studies fall short of a systematic identification of all possible structures and control configurations.

In order to facilitate design and choice of appropriate paralleling configurations, a systematic identification of the paralleling schemes that permits a clear exposure of the structures, behaviors and limitations of all possible schemes, is needed. In this paper, we investigate the general problems for paralleling sources and utilize basic circuit theory to identify the basic structures and control methods of paralleled dc/dc converters. Characteristics for the various paralleling schemes will be studied according to the circuit theoretic identification. Our objective is to provide a clear set of design guidelines for engineers who have to take into account the practical requirements of the system to be designed.

Our starting point will be the two Kirchhoff's laws that

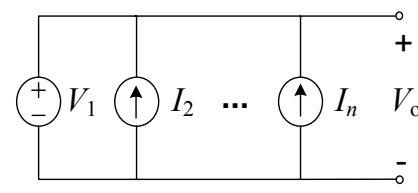

(a)

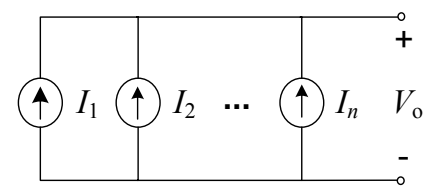

(b)
Fig. 1. Structures for paralleling ideal independent sources.

dictate the possible connection styles [3]. Considering converters as either voltage sources or current sources, we define three basic structures for paralleling converters. As we will see, these structures actually form the basis of all practical paralleling schemes. We will develop equivalent models which can be used in analysis. Moreover, control methods will be systematically introduced to complete the output regulation and current-sharing functions. Furthermore, a set of experimental prototype which consists of two parallel-connected buck converters and six different controllers, are constructed to validate the behavior of the recognized basic schemes. Finally, the dynamic and steady-state performances of the basic schemes are compared experimentally.

\section{BASic Circuit Theory of PARALlel Connections}

Two basic laws must be obeyed when connecting sources together. First, Kirchhoff's voltage law (KVL) dictates that no two independent voltage sources are permitted to be connected in parallel. Theoretically, even if the voltage sources are of the same magnitude, paralleling them is still not permitted as it makes the current values undefined [4]. Likewise, Kirchhoff's current law (KCL) eliminates the possibility of connecting two independent current sources in series. In this paper, as our focus is paralleling sources, we do not consider the case of connecting sources in series.

From the above discussion, it is clear that independent sources can be connected in parallel under only two possible circumstances, as shown in Fig. 1. First, only one of them can be an independent voltage source, and the rest must be current sources, as shown in Fig. 1 (a). The output voltage is decided by the voltage source branch, and the current in the voltage source is determined by the load. Second, all parallel branches are current sources, as shown in Fig. 1 (b). The output voltage is decided by the load.

It should be clear that in practice, the voltage and current 


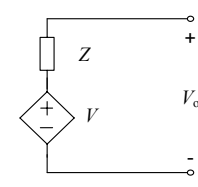

(a)

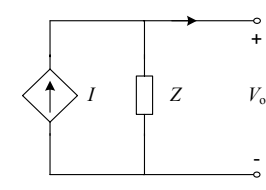

(b)
Fig. 2. Equivalent circuits for power converters. (a) Thévenin form; (b) Norton form.

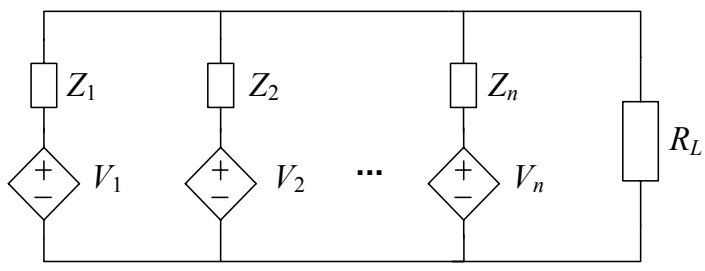

(a)

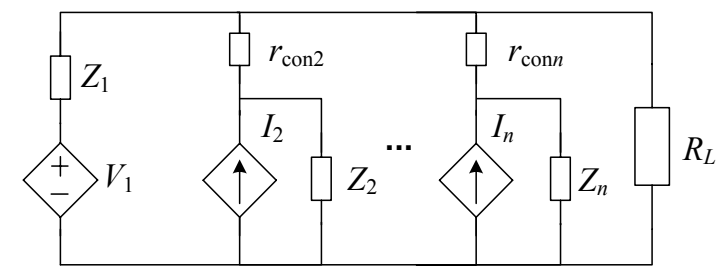

(b)

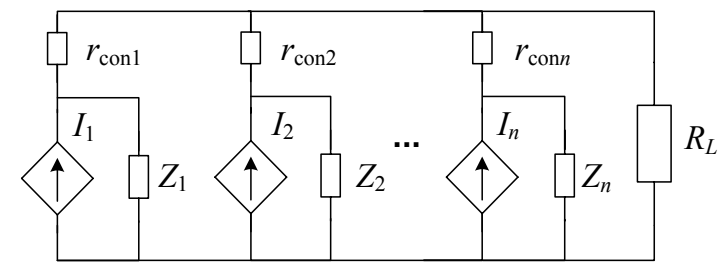

(c)

Fig. 3. Three configurations for paralleling converters. (a) Type I; (b) Type II; (c) Type III.

sources are not independent but are controlled sources in order to allow regulated output voltage and specific sharing of current to be maintained. In general, we may simply and generically represent a dc/dc converter in Thévenin form or Norton form, i.e., a dependent voltage behind a small impedance (at low frequency) or a dependent current source in parallel with a large impedance, as shown in Fig. 2.

\section{General Classification of Parallel CONNECTED DC/DC CONVERTERS}

From the foregoing discussion, it is clear that any paralleling scheme involving voltage and current sources must comply with the two basic structures described earlier. Moreover, if the voltage sources are imperfect, ${ }^{1}$ they can still be connected in parallel. Thus, we have three basic configurations for paralleling imperfect sources.

When dc/dc converters are treated as imperfect voltage or current sources, three basic configurations for paralleling practical dc/dc converters can be developed, as summarized in

\footnotetext{
${ }^{1}$ By imperfect sources, we mean those voltage sources having non-zero output impedance and those current sources having finite output impedance.
}

Fig. 3. For brevity, we refer to these configurations as Types I, II and III connections. For a voltage source branch, we have

$$
V_{o}=V_{i}-I_{o, i} Z_{i} \quad \text { or } \quad I_{o, i}=\frac{V_{i}-V_{o}}{Z_{i}}
$$

where subscript $i$ ( 1 to $n$ ) indicates the branch number, and $I_{o, i}$ is the output current of the $i$ th branch, i.e., the part of load current shared by the $i$ th branch. For a current source branch, we have

$$
V_{o}=\left(I_{i}-I_{o, i}\right) Z_{i} \quad \text { or } \quad I_{o, i}=I_{i}-\frac{V_{o}}{Z_{i}}
$$

where $I_{i}$ is the equivalent current source of the $i$ th branch.

In practice, we need to apply appropriate control to dc/dc converters in order to "cast" them as voltage or current sources. For instance, a voltage feedback loop is obviously needed for controlling a dc/dc converter so that it behaves as a voltage source. Thus, the paralleling configurations are closely related to the control method which effectively determines whether a $\mathrm{dc} / \mathrm{dc}$ converter would behave as a voltage or current source.

In addition to the defining control of current and voltage sources, a current-sharing control can be used to ensure even sharing of the load among the converters. To avoid confusion, we will use the term current-sharing loop in a specific context. If a current-sharing control signal is derived from the output currents of one/all constituent converters, the control scheme is said to contain a current-sharing loop. Otherwise, the control scheme does not have a current-sharing loop.

We may therefore further classify parallel converter systems according to the presence of a current-sharing loop, resulting in a simple, systematic classification, as shown in Fig. 4. Two layers are included in the classification. In the first layer, we get three configurations, Types I, II and III, based on the circuit theoretic connection styles. In the second layer, the presence of a current-sharing loop is the classifying criterion.

\section{Three Types of Connection Styles And Associated CONTROL Methods}

In this section, in light of the classification framework mentioned in the foregoing, the various types of parallel connected $\mathrm{dc} / \mathrm{dc}$ converters are described in detail. Our emphases here are the generic circuit theoretic structures and the necessary control methods. As a prerequisite, we note that converters aiming to imitate voltage sources should have tight voltage feedback loops for voltage regulation purposes, whereas converters imitating current sources would necessitate some form of current-mode control in order to set the current magnitudes. The presence of a current-sharing loop is an additional feature, contributing to the current sharing of the constituent converters.

\section{A. Type I}

The Type I connection is shown in Fig. 3 (a). Each branch represents a converter, which is basically a Thévevin source. For the control without a current-sharing loop, the branches are simply connected in parallel. No other extra action is taken among the converters to achieve current balance. However, 


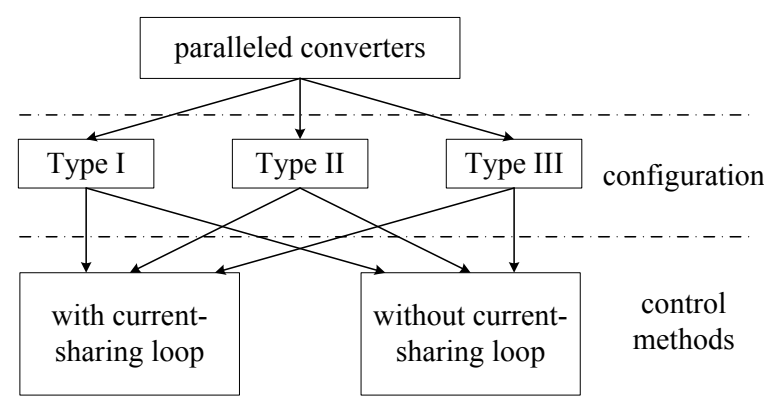

Fig. 4. A systematic classification of parallel connected converters.

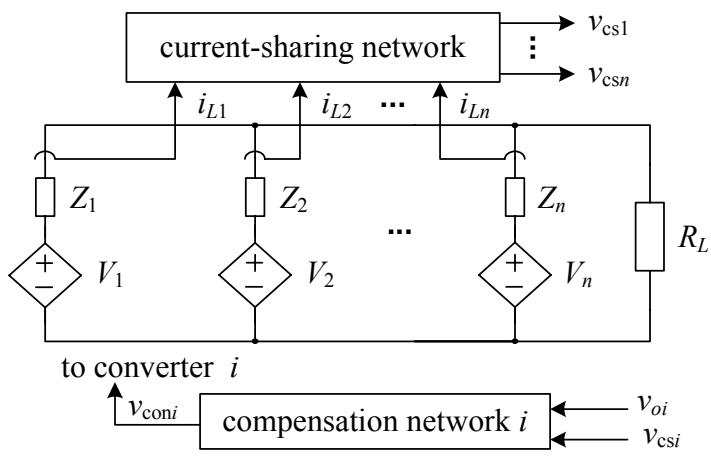

Fig. 5. Control structure for Type I configuration with a current-sharing loop.

the absence of a current-sharing loop imposes some specific requirements on the individual branches in order to provide natural current sharing. This has been commonly known as the droop method [1]. Specifically, each converter, in the absence of a current-sharing loop, should have a finite output resistance at steady state, which results in obvious droop characteristic of the converter. Otherwise, any small discrepancy of $V_{i}$ and/or $Z_{i}$ will cause severe current imbalance among the converters.

For Type I connection with a current-sharing loop, since all converters are Thévenin sources, output regulation and current sharing are achieved by controlling $V_{1}, V_{2}, \cdots, V_{n}$ and/or the output impedance $Z_{1}, Z_{2}, \cdots, Z_{n}$. The control structure is shown in Fig. 5. In this configuration, each converter is a dependant voltage source, whose output voltage is controlled directly. The currents sensed from different converters are programmed to obtain a common current-sharing control signal, which will be compared with the feedback currents to regulate individual equivalent voltages $V_{1}, V_{2}, \cdots, V_{n}$. The objective is to shrink the discrepancy of the converters [5]. Thus, all converters share the load equally.

\section{B. Type II}

For the Type II connection shown in Fig. 3 (b), one converter serves as the voltage (Thévenin) source and others are current (Norton) sources. The control structure without a current-sharing loop is shown in Fig. 6 (a). There is a main voltage feedback loop, which acts on the voltage (Thévenin) source to regulate the output voltage. Other branches are under current-mode control (peak-current-mode control is applied in the paper), whose objective is to make all individual output

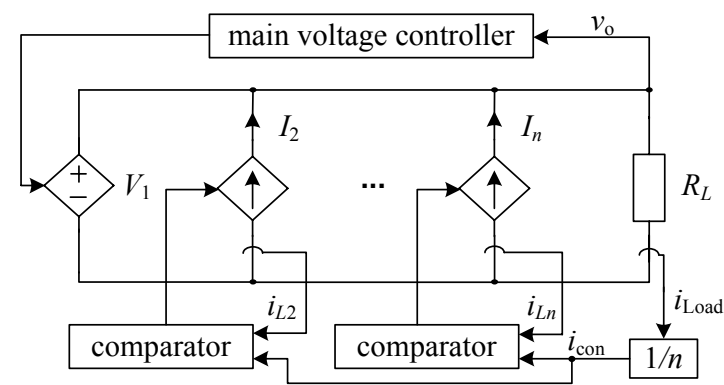

(a)

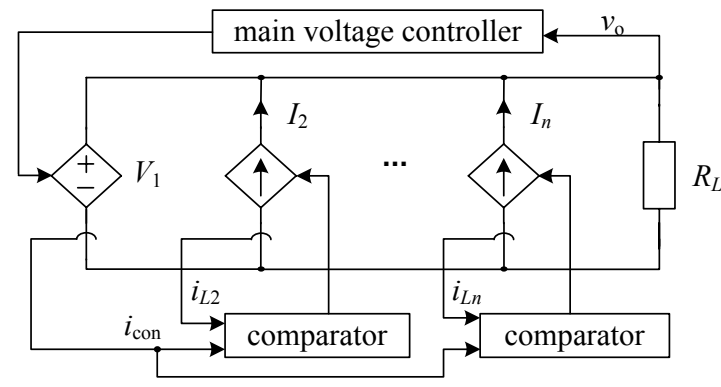

(b)

Fig. 6. Control structures for Type II configuration (a) without a currentsharing loop; (b) with a current-sharing loop.

currents share the same portion of the load current.

For the Type II configuration with a current-sharing loop, the control structure is shown in Fig. 6 (b). Again, there is a main voltage loop to control the voltage source. The current control signal for the current sources will be derived from the voltage source branch. This current control signal is then compared with the individual current of the $n-1$ converters to achieve current sharing. This method is commonly known as master-slave current-sharing method [1], where the voltage source is the master and the current sources are the slaves whose currents are programmed to follow the master's.

\section{Type III}

In the Type III configuration shown in Fig. 3 (c), all converters are current (Norton) sources. In the absence of a current-sharing loop, all converters have to follow a currentsharing control signal which is derived from the output voltage feedback loop, as shown in Fig. 7 (a). The feedback loop aims to achieve voltage regulation as well as current sharing.

Finally, for the Type III configuration with a current-sharing loop, all converters are under current-mode control so that they behave as good current sources. Current-programming methods, such as master-slave method or average method, can be used to generate the common current-sharing control signal. The amplified errors between the current-sharing control signal and the feedback currents are injected to the feedback loop, as shown in Fig. 7 (b) [6].

\section{Comparison of Basic Paralleling Schemes}

From the structures and the associated control methods for paralleling $\mathrm{dc} / \mathrm{dc}$ converters, intuitively, we can make the following general observations. 


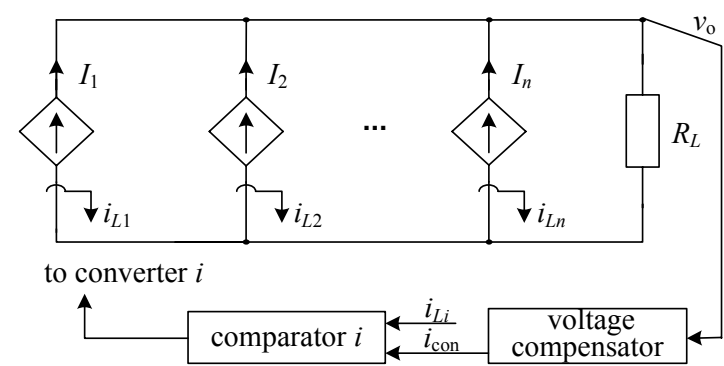

(a)

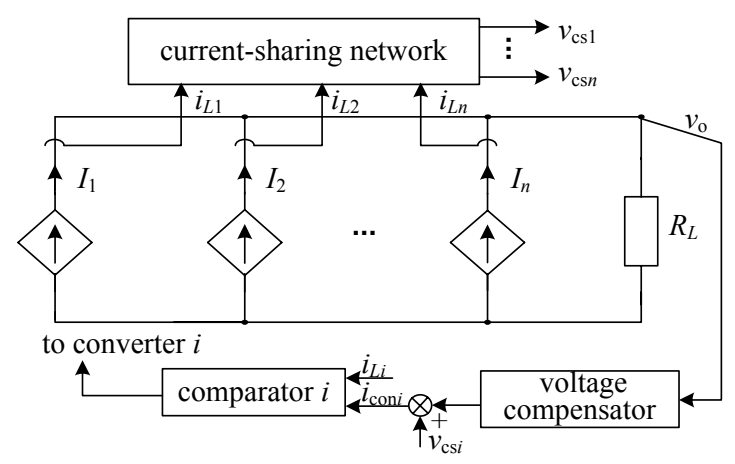

(b)

Fig. 7. Control structures for Type III configuration (a) without a currentsharing loop; (b) with a current-sharing loop.

1) Type I schemes are simple but suffer fundamentally from the connection of paralleling voltage sources. The adjustment range for current sharing is small since each constituent converter is designed primarily to regulate its output voltage. Current sharing is achieved by adjusting the output characteristics of the constituent converters.

2) Type II schemes are theoretically more viable as there is only one voltage source paralleling with current sources. The dynamics of the voltage regulation thus depends on the control method being employed by the voltage regulating loop. The other current source converters control their currents directly to achieve the desired current sharing. Thus, the current-sharing performance is generally much better and the control implementation is simpler, compared to Type I schemes.

3) Type III schemes are generally best in terms of current sharing as all converters are fundamentally current controlled. The voltage regulation is only executed at the load side. Both voltage regulation and current sharing are excellent.

\section{EXPERIMENTAL VERIFICATIONS}

In the foregoing section, we have discussed the operating principles and performances of the different connections and control schemes for paralleling dc/dc converters. Some qualitative comparisons are made based on intuitive analysis. To validate the analysis, a set of prototype consisting of two buck converters $(24 / 12 \mathrm{~V}, 8 \mathrm{~A})$ connected in parallel has been constructed. Six different controllers, as explained in the previous section, are designed and constructed to compare the

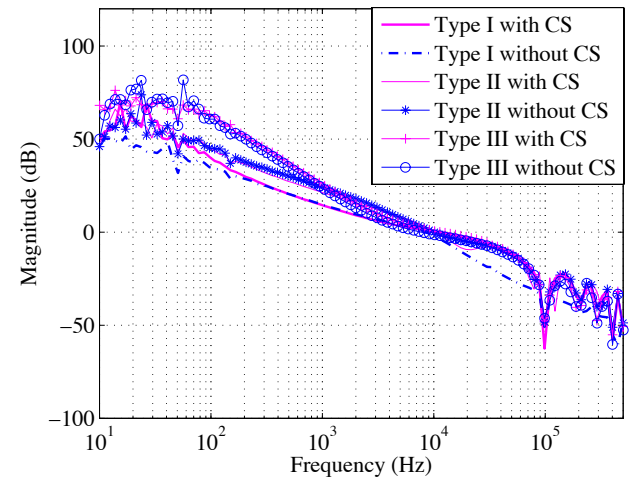

Fig. 8. Measured voltage-loop gains for the six schemes.

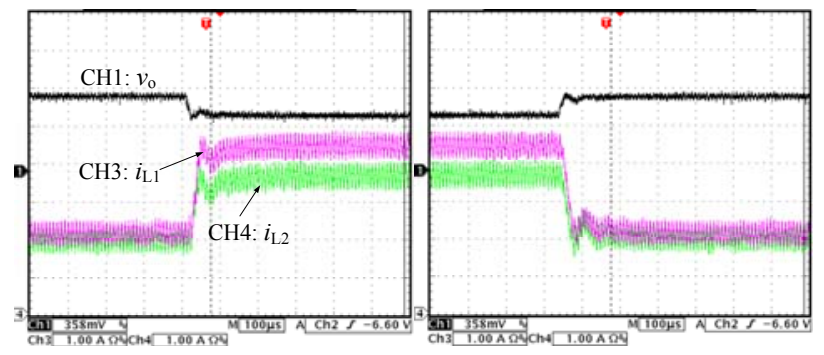

(a)

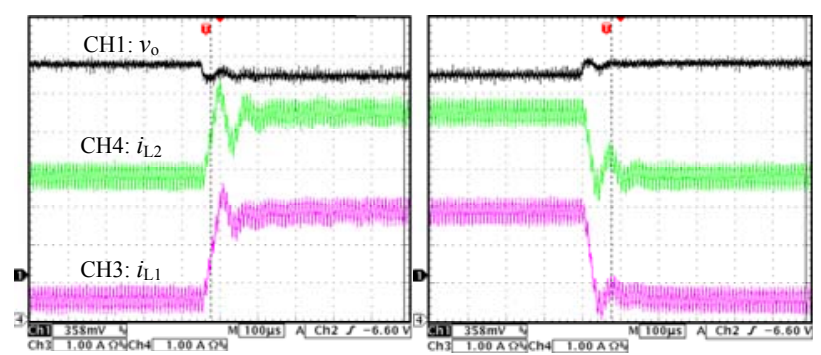

(b)

Fig. 9. Stepped load results of Type I scheme without a current-sharing loop at $V_{\text {ref1 }}=V_{\text {ref2 }}=12 \mathrm{~V}, r_{\operatorname{con} 1}=0.001 \Omega, r_{\text {con2 }}=0.05 \Omega$. (a) with large output impedance; (b) with small output impedance.

performances of all the schemes. To ensure validity of the comparisons, the bandwidth of voltage loops are set at $10 \mathrm{kHz}$ for all the schemes, as shown in Fig. 8. The comparisons are made in terms of the performances in current sharing and voltage regulation.

In the configuration of Type I, we fix both converters' bandwidth at $10 \mathrm{kHz}$ to ensure the same transient capability of voltage regulation. For the control without a current-sharing loop, we introduce a current feedback to control the output impedance. The experiment results for a stepped load test is shown in Fig. 9. As shown in Fig. 9 (a), reasonable current sharing is observed at half load. However, current sharing becomes worse at full load. Furthermore, we observe a large output voltage droop after the transient. Figure 9 (b) shows the dynamic response for smaller output impedance where we observe poorer current sharing but smaller voltage droop. The output droop characteristic is displayed in Fig. 10 by collecting 


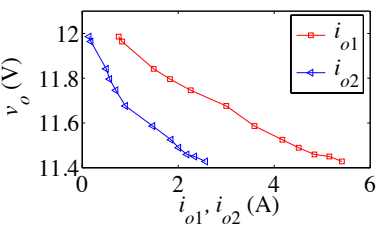

(a)

(c)

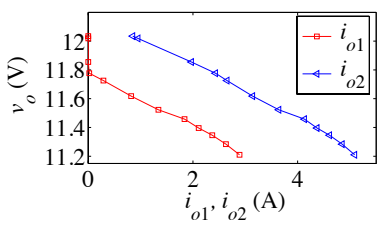

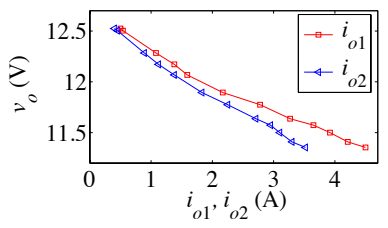

(b)

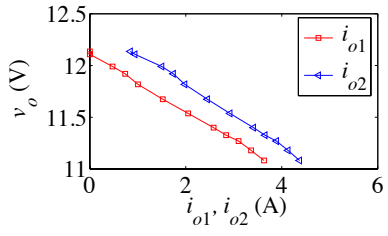

(d)
Fig. 10. Output voltage versus output current for Type I scheme without a current-sharing loop. (a) $V_{\text {ref1 }}=V_{\text {ref2 }}=12 \mathrm{~V}, r_{\text {con } 1}=0.001 \Omega, r_{\text {con2 }}=$ $0.05 \Omega$, with small output impedance; (b) $V_{\text {ref1 }}=V_{\text {ref2 }}=12 \mathrm{~V}, r_{\operatorname{con} 1}=$ $0.001 \Omega, r_{\operatorname{con} 2}=0.05 \Omega$, with large output impedance; (c) $V_{\text {ref1 }}=11.5$ $\mathrm{V}, V_{\text {ref2 }}=12 \mathrm{~V}, r_{\operatorname{con} 1}=r_{\operatorname{con} 2}=0.025 \Omega$, with small output impedance; (d) $V_{\text {ref1 }}=V_{\text {ref2 }}=12 \mathrm{~V}, r_{\text {con1 }}=r_{\text {con2 }}=0.025 \Omega$, with large output impedance.
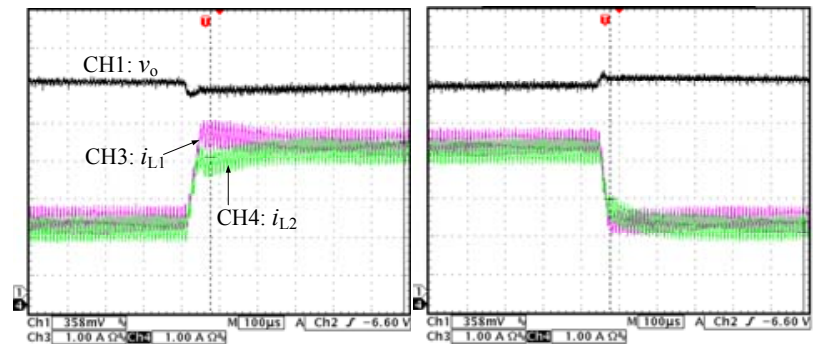

Fig. 11. Stepped load results of Type I scheme with a current-sharing loop at $V_{\text {ref1 }}=V_{\text {ref2 }}=12 \mathrm{~V}, r_{\mathrm{con} 1}=0.001 \Omega, r_{\mathrm{con} 2}=0.05 \Omega$.

the steady-state voltage and currents. Specifically, Fig. 10 (a) shows the results of the two converters with the same reference output voltage, but different connection resistors. Figure 10 (c) corresponds to the case of two converters with the same connection resistors, but different reference output voltages. Likewise, Figs. 10 (b) and (d) give the corresponding results when larger output impedances are used. All the results are collected under the assumption of $10 \mathrm{kHz}$ bandwidth for the voltage loops. From the experimental results, we clearly see that the configuration without a current-sharing loop does not perform very satisfactorily. Normally, with large output impedance, we may achieve good current sharing but poor output regulation. However, the current sharing becomes worse and output regulation becomes better with smaller output impedance. In practice, we have to trade off voltage regulation for current sharing.

For the connection of Type I with a current-sharing loop, the bandwidth of the current-sharing loop is one-tenth of the voltage loop's. The results are much better than the case without a current-sharing loop, as demonstrated in Figs. 11 and 12. Figure 11 shows the output voltage and inductor currents under a stepped load test. The current error is very small and so does the voltage droop. Figure 12 (a) shows the

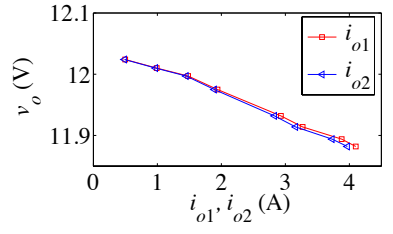

(a)

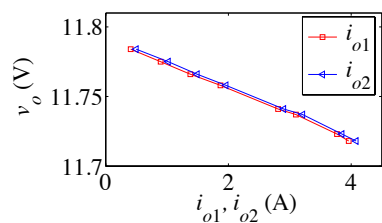

(b)
Fig. 12. Output voltage versus output current for Type I scheme with a current-sharing loop. (a) $V_{\text {ref1 }}=V_{\text {ref2 }}=12 \mathrm{~V}, r_{\operatorname{con} 1}=0.001 \Omega, r_{\text {con2 }}=$ $0.05 \Omega$; (b) $V_{\text {ref1 }}=11.5 \mathrm{~V}, V_{\text {ref } 2}=12 \mathrm{~V}, r_{\operatorname{con} 1}=r_{\operatorname{con} 2}=0.001 \Omega$.

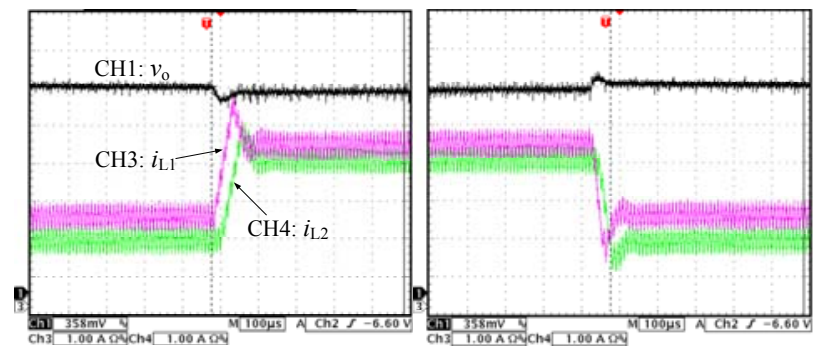

(a)

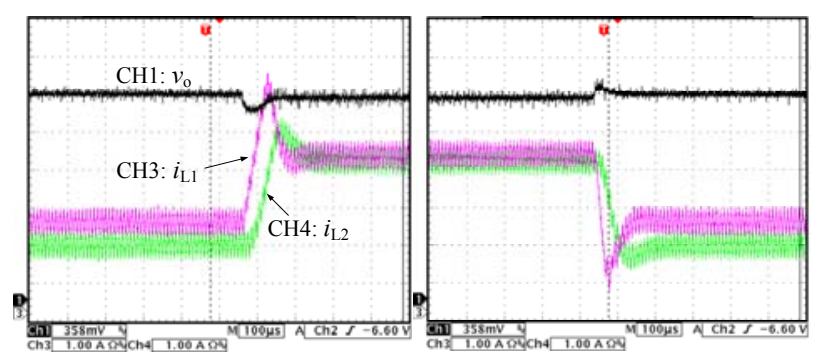

(b)

Fig. 13. Stepped load results for Type II scheme at $V_{\text {ref }}=12 \mathrm{~V}, r_{\mathrm{con} 1}=$ $0.001 \Omega, r_{\operatorname{con} 2}=0.05 \Omega$, (a) without a current-sharing loop; (b) with a current-sharing loop.

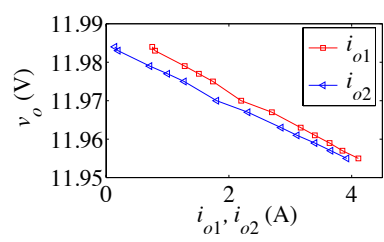

(a)

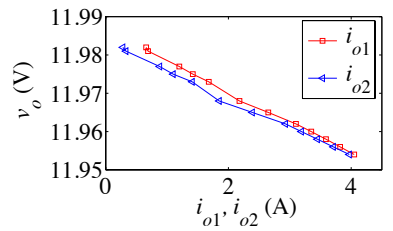

(b)
Fig. 14. Output voltage versus output current for Type II scheme at $V_{\text {ref }}=$ $12 \mathrm{~V}, r_{\mathrm{con} 1}=0.001 \Omega, r_{\operatorname{con} 2}=0.05 \Omega$, (a) without a current-sharing loop; (b) with a current-sharing loop.

steady-state performance of the two converters under the same reference output voltage, but different connection resistors and Fig. 12 (b) shows the results of the two converters with the same connection resistors, but different reference output voltages. Here, we observe that the current sharing result is good when the converters have the same reference output voltage. However, there is a constant current error if the reference output voltages are different, as shown in Fig. 12 (b). This is because the current-sharing loop can regulate the equivalent output impedance, but cannot remove the inherent 


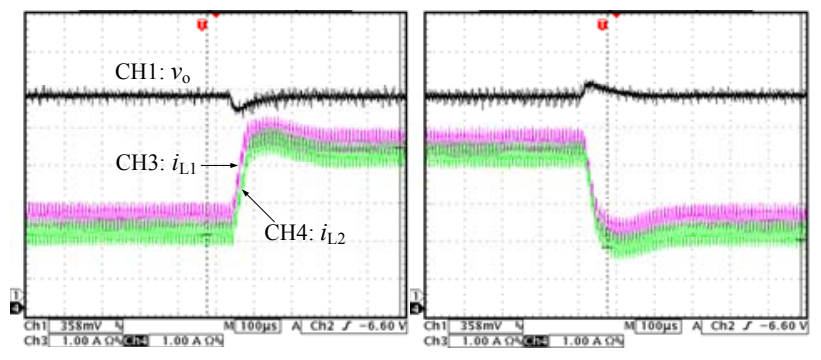

(a)

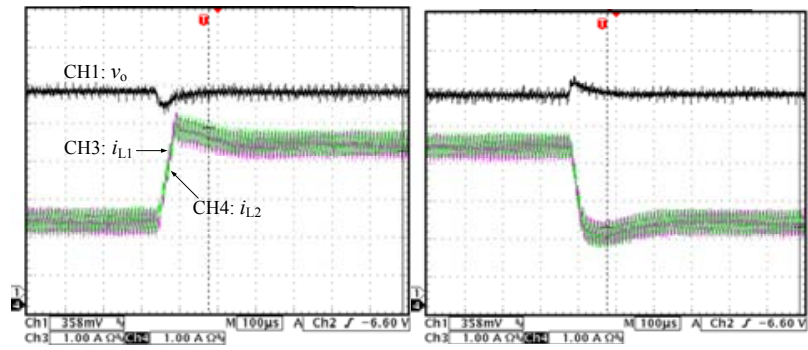

(b)

Fig. 15. Stepped load results for Type III scheme at $V_{\text {ref }}=12 \mathrm{~V}, r_{\mathrm{con} 1}=$ $0.001 \Omega, r_{\operatorname{con} 2}=0.05 \Omega$, (a) without a current-sharing loop; (b) with a current-sharing loop.

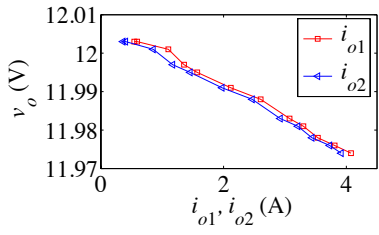

(a)

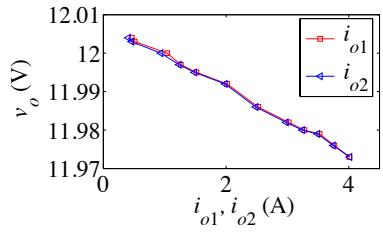

(b)
Fig. 16. Output voltage versus output current for Type III scheme at $V_{\text {ref }}=$ $12 \mathrm{~V}, r_{\mathrm{con} 1}=0.001 \Omega, r_{\operatorname{con} 2}=0.05 \Omega$, (a) without a current-sharing loop; (b) with a current-sharing loop.

voltage difference between the converters.

Shown in Figs. 13 and 14 are the experimental results for Type II configuration with and without a current-sharing loop. The transient responses for both cases are similar since their only difference is the derivation of the current control signal for the Norton source. In Fig. 13, the large current-sharing error is observed at load transient, which is the result of the use of different control modes for Thévenin source and Norton source. Better current-sharing performance can be achieved with the use of a current-sharing loop, especially at heavy load. From the steady-state performance under different loads shown in Figs. 14 (a) and (b), we notice that the voltage droop is much smaller than that of paralleling Thévenin sources because of the tight regulation of the load voltage. Also, the output voltage will not be affected by the connection resistor $r_{\text {con } i}$. In Fig. 14, we observe almost constant current difference in the whole load range. This can be attributed to the use of peak currentmode control. Here, the current control signal of the Norton source is derived from the average current of the master/the load. As a result, there is a constant current difference between the converters, which is equal to half of the current ripple. A biased voltage may be introduced to reduce such an error.
For Type III configuration, only one voltage loop is applied at the load side. For the control with a current-sharing loop, again, the bandwidth of the current-sharing loop is one-tenth of the voltage loop's. Figures 15 and 16 show the performance under a stepped load and steady-state test, respectively. In the figures, we observe no noticeable difference in the voltageregulation performance for the cases with and without a current-sharing loop. However, in terms of current-sharing performance, the case with a current-sharing loop is significantly better. From Fig. 16, nearly perfect load voltage regulation and current sharing for both cases with and without a currentsharing loop are observed. The current error is much smaller compared to the other schemes. Even for the control without a current-sharing loop, the current sharing is superior since all the converters are under current-mode control and follow the same control signal.

\section{CONCLUSION}

In this paper, the classification and control of parallel-connected $\mathrm{dc} / \mathrm{dc}$ converters are studies from a circuit theoretic viewpoint. Three basic types of paralleling schemes can be identified based on circuit theory, namely Types I, II and III configurations. Then, control methods with and without a current-sharing loop to achieve both voltage regulation and current sharing are detailed. For Type I configuration, each converter has its own voltage loop. Obvious droop characteristic is observed for both schemes with and without a current-sharing loop. In the case where a current-sharing loop is absent, we have to trade off voltage regulation for current sharing, whereas in the case where a current-sharing loop is present, the role of the current-sharing loop is to regulate the output characteristic of the constituent converters. For Type II configuration, the control method is much simpler than that of Type I connection, and the accuracy of current sharing can be further improved by proper design of the current source controllers. In the case of Type III configuration, voltage regulation is executed at the load side. Each converter only needs to follow a current control signal. Both control methods perform well. The role of the currentsharing loop is to regulate the current control signal directly.

Finally, it should be reiterated that our study does not pinpoint a particular scheme as being the preferred or unpreferred scheme. The choice is up to the designers who have to take into account the practical requirements of the system to be designed. Our purpose here is to assist the designers in making this choice.

\section{ACKNOWLEDGMENT}

This work is supported by Hong Kong Polytechnic University under project G-U284.

\section{REFERENCES}

[1] S. Luo, Z. Ye, R.-L. Lin, and F. C. Lee, "A classification and evaluation of paralleling methods for power supply modules," Proc. IEEE Power Electron. Specialists Conf. Record, pp. 901-908, June 1999.

[2] B. Choi, "Comparative study on paralleling schemes of converter modules for distributed power applications," IEEE Trans. on Ind. Electronics, vol. 45, no. 2, pp. 194-199, March 1998.

[3] Y. Huang and C. K. Tse, "Circuit theoretic classification of parallel connected DC-DC converters," IEEE Trans. Circ. Syst. Part I, vol. 54, no. 5, pp. 1099-1108, May 2007.

[4] C. K. Tse, Linear Circuit Analysis, London, UK: Addison Wesley, 1998.

[5] Y. Panov and M. M. Jovanovic, "Stability and dynamic performance of current-sharing control for paralleled voltage regulator modules," IEEE Trans. Power Electron., vol. 17, no. 2, pp. 172-179, March 2002.

[6] C.-S. Lin and C.-L. Chen, "Single-wire current-share paralleling of current-mode-controlled DC power supplies," IEEE Trans. Industrial Electron., vol. 47, no. 4, pp. 780-786, April 2000. 